\title{
Novel fractional bipolar radiofrequency device for the treatment of aged Asian skin.
}

\author{
Lifang Guo", Rong Zeng", Tong Lin*, Yuqing Huang, Yuzhen Liu \\ Institute of Dermatology, Chinese Academy of Medical Sciences and Peking Union Medical College, Cosmetic Lasers, \\ Nanjing, PR China \\ \#These authors have equally contributed to this work.
}

\begin{abstract}
Background and objective: Selecting an ideal method for restoring youthful skin among Asian patients is complicated because of their intrinsic skin color. Bipolar fractional radiofrequency (FRF) gains increasing attention for acne scar treatment in Asian patients. Nevertheless, clinical experience on FRF treatment of aged Asian skin remains limited. Our study aims to assess the efficacy and safety of FRF and tolerance against in aged Asian skin.

Methods: Patients with wrinkles on different regions (forehead=8, lateral canthus=10, $c=12$, and neck=12) received three treatments $(50-60 \mathrm{~mJ} / \mathrm{pin})$ with a bipolar FRF device Digital photographs, improvement and tolerance of the patients were taken at each visit.

Results: The wrinkling scale significantly decreased in each type of wrinkles after 2 and 3-months of follow-up $(\mathbf{P}<\mathbf{0 . 0 5})$. The satisfaction of the patients with regard to the treatment of their wrinkles was consistent with the findings of the investigator. Side effects, such as dryness, transient erythema, pain were detected. All these symptoms are tolerable.

Conclusions: FRF device is an effective and safe treatment for skin rejuvenation for aged Asian.
\end{abstract}

Keywords: Fractional bipolar radiofrequency, Asian patient, Photo aging, Wrinkles.

Accepted on July 01, 2018

\section{Introduction}

Coarse wrinkles, laxity, rough texture, dyspigmentation, and telangiectasia are symptoms of facial aging. Lasers, intense pulsed light, and radiofrequency (RF) technologies have been utilized for skin rejuvenation over the past few years. Among laser treatments, ablative laser technology is conventionally used for skin rejuvenation; this technique exhibits robust efficacy but presents considerable downtime and risk for scarring and hyperpigmentation. By contrast, the use of nonablative lasers has limited effectiveness but can greatly reduce downtime. For treatment of Asian skin, laser-based technologies present several limitations, particularly diffraction, chromophore absorption, and scattering, which could reduce the amount of energy targeted at the dermis and increase the risk for hyperpigmentation [1]. These constraints are not observed in RF, which produces electric current rather than light [2]. Thus, RF-based systems are regarded suitable for all skin types, such as Asian skin [3].

RF technology has been introduced for photo-aging treatment in Asians with dark skin types [4]. This technique utilizes a frequency range of $3 \mathrm{kHz}$ to $300 \mathrm{GHz}$ non-ionizing electromagnetic radiation [5]. Fractional photothermolysis (FP) has been successfully used in the field of laser photo-aging therapy [6]. This modern technique enables the laser to form an array of microscopic thermal damage on the skin, thereby leaving the untreated skin as a reservoir of viable tissues and reducing the risk for complications [7,8]. The first bipolar fractional RF device was developed in 2009 [9]; since then, this technology has been used for skin rejuvenation in Caucasian patients [9-14]. Nevertheless, clinical experience of this technology for treatment of aged Asian skin remains limited. In present study, we used a FRF device to treat Asian skin photo-aging and evaluated the efficacy and safety of this technology.

\section{Materials and Methods}

\section{Patients}

43 Chinese patients (age range from 30 to 60, Fitzpatrick skin types IV-V) with aged and/or mild rhytids and photo-damaged skin were recruited in the Institute of Dermatology, Chinese Academy of Medical Sciences. The exclusion criteria included pregnancy or lactation, infection, laser or other cosmetic treatments within the last 6 months, HIV status, history of keloid and photosensitivity disorders, compromised immunity and intake of oral retinoids within the past 12 months. All the 
patients in the group conformed to the guideline of Helsinki convention. The authors have no conflicts of interest.

\section{Equipment}

A bipolar fractionated RF device (eMatrix ${ }^{\mathrm{TM}}$, Syneron Medical Ltd.) was used. A hand-held applicator was equipped with a small square, which consists of 64-electrode-pin disposable tips per $12 \mathrm{~mm} \times 12 \mathrm{~mm}$. During the entire treatment, the tips were vertically positioned over the skin, and the treated area was completely dried. The treatment parameters were based on the pre-existing skin conditions, lesion distribution, specific anatomical location, and pain tolerance. The distribution of lesions, specific anatomical local, the pre-existing skin conditions and pain tolerance are considerations for treatment parameters. Prior to the study, we performed and evaluated different programs with this device for treatment of several patients. As such, in the present study, we set the final parameters for wrinkles on different regions as follows: forehead (55-60 $\mathrm{mj} / \mathrm{pin})$, lateral canthus (50-55 $\mathrm{mj} / \mathrm{pin})$, glabellum (50-60 mj/pin), and neck (50-60 mj/pin). A doublepass treatment was performed, with $5 \%$ or $10 \%$ coverage rate.

During the first visit, the subject was evaluated to determine the appropriate treatment site and received three treatments on the corresponding site every 4 to 6 weeks. There is no need to use any local anesthesia before or during treatment. An iced pad was used after the treatment on the treated area. We recommend the patients to use moisturizer and sunscreen regularly during the study.

\section{Outcome assessments}

Assessments were performed at three time points over the study period (at baseline before treatment (visit 1), 4 weeks after the second treatments (visit 3), and 4 weeks after the final (third) treatment (visit 4)). During each visit, frontal, $45^{\circ}$ and $90^{\circ}$ photographs of the neck and face were taken.

\section{Objective evaluation}

Outcomes were assessed at the time mentioned above. Photographic documentations were obtained. Three experienced dermatologists blindly assessed the clinical improvement by comparing pre-treatment and post-treatment photographs. Wrinkles on the face and neck were graded into three levels (mild, moderate, and severe.) according to the guidelines of the Japanese Cosmetic Science Society. The scores are presented in Table 1.

Table 1. The wrinkle score modified from the guideline of wrinkle from Japanese Cosmetic Science Society. Each grading of wrinkles was classified as: (i) Mild; (ii) Moderate; (iii) Sever and subsequently scored.

\begin{tabular}{llll}
\hline & Mild & Moderate & Severe \\
\hline Grade 0 & 0 & 0 & 0 \\
\hline Grade 1 & 1 & 2 & 3
\end{tabular}

\begin{tabular}{llll}
\hline Grade 2 & 4 & 5 & 6 \\
\hline Grade 3 & 7 & 8 & 9 \\
\hline Grade 4 & 10 & 11 & 12 \\
\hline Grade 5 & 13 & 14 & 15 \\
\hline Grade 6 & 16 & 17 & 18 \\
\hline Grade 7 & 19 & 20 & 21 \\
\hline
\end{tabular}

Wrinkle improvement was assessed by the investigator by using a six-point scale.

\section{Subjective evaluation}

Patients were asked to document their own assessment of wrinkles on the forehead, glabellum, lateral canthus, and neck. A 10-point visual analog scale (VAS) was used to assess the level of pain ( 0 indicating no pain and 10 signifying worst pain). Patients were also asked to provide information on side effects, such as edema, erythema, ulceration, pigmented alteration, and infection.

The data were analyzed using SPSS software. Student's t-test was used for comparison between visits. Data are presented as mean \pm standard deviations $(\mathrm{SD})$, and $\mathrm{P}<0.05$ was considered statistically significant.

\section{Results}

Among the subjects enrolled in this study, 42 patients (mean age, $43.04 \pm 9.68$; Fitzpatrick skin type IV and V) completed the study and one patient was excluded because of failure to return for follow-up visits.

\section{Objective evaluation}

Based on the established evaluation rules for wrinkle improvement, almost all of the subjects with different types of wrinkles manifested clinical improvement after three treatments (Figures 1 and 2). Figure 2A shows that the mean investigator improvement scores for wrinkles on the forehead at the follow-up visit exceeded that at the baseline $(14.125 \pm$ $6.08)$, with $9.75 \pm 5.75(\mathrm{P}<0.05)$ at visit 3 and $9.25 \pm 5.15$ $(\mathrm{P}<0.05)$ at visit 4 . Figure $2 \mathrm{~B}$ shows that the mean investigator improvement scores for wrinkles on the lateral canthus at the follow-up visit exceeded that at the baseline $(10.7 \pm 5.66)$, with $6.6 \pm 4.42(\mathrm{P}<0.05)$ at visit 3 and $6.1 \pm 4.65(\mathrm{P}<0.05)$ at visit 4. Figure $2 \mathrm{C}$ shows that the mean investigator improvement scores for wrinkles on the glabellum at the follow-up visit exceeded that at the baseline $(11.75 \pm 5.55)$, with $7.08 \pm 3.99$ $(\mathrm{P}<0.05)$ at visit 3 and $6.5 \pm 4.27(\mathrm{P}<0.05)$ at visit 4. Figure $2 \mathrm{D}$ shows that the mean investigator improvement scores for wrinkles on the neck at the follow-up visit exceeded that at the baseline $(13.92 \pm 3.58)$, with $7.25 \pm 2.09(\mathrm{P}<0.05)$ at visit 3 and $6.42 \pm 1.83(\mathrm{P}<0.05)$ at visit 4 . These results indicate that fractional bipolar RF device can improve wrinkles on forehead, lateral canthus, glabellum, and neck. 

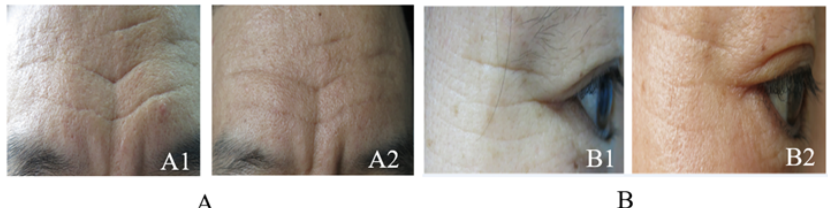

Forehead

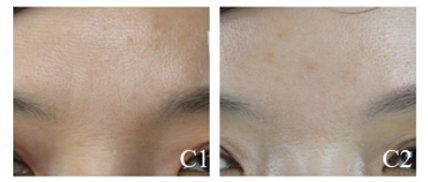

$$
\text { C }
$$

Glabellum

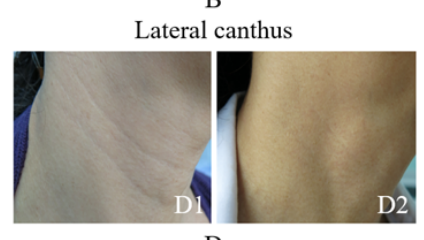

D

Figure 1. Representative photographs of four subjects showing improvements after three fractional biopolar radiofrequence (RF) treatments. (A) Forehead; (B) Lateral canthus; (C) Glabellum; (D) Neck; (A1, B1, C1, D1) pre-treatment; $(A 2, B 2, C 2, D 2)$ four weeks after the third treatment.
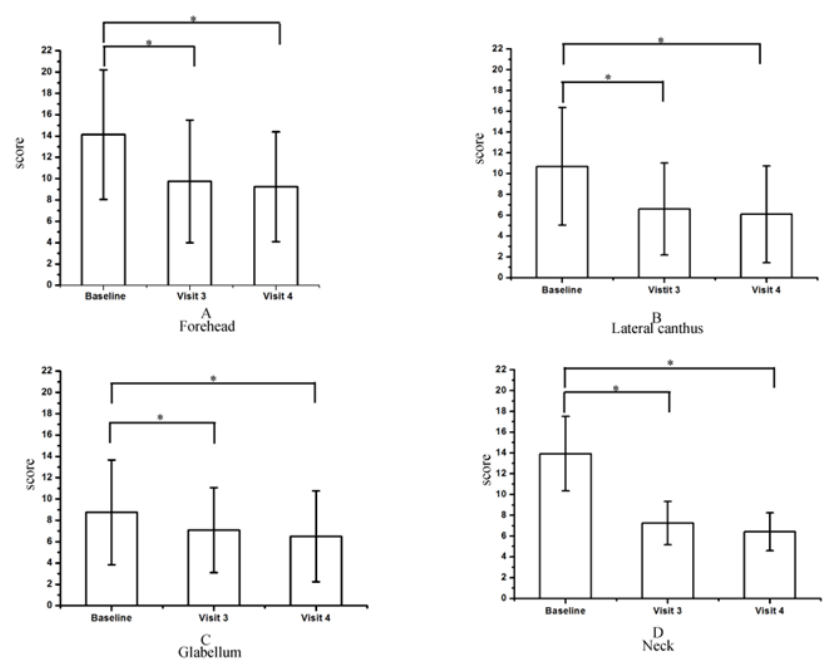

Figure 2. (A-D) The comparison average scores between baseline and visit 3 (4 weeks after two sessions, before irradiation) or visit 4 (4 weeks after the last treatment, before irradiation). The difference in the average wrinkle scores of forehead, lateral canthus, glabellum and neck between baseline and visit 3/4 was statistically significant $\left({ }^{*} p<0.05\right)$.

In patients with wrinkles on the forehead, only one subject reported no changes after three treatments, and the seven other subjects indicated moderate to very good improvement (Figure 3). In patients with wrinkles on the glabellum, two subjects reported no change after three treatments, and the eight other subjects indicated slight to very good improvement at visit 4 . In patients with wrinkles on the lateral canthus and neck, all subjects indicated slight to very good improvement at visit 4 .

\section{Subjective evaluation}

The subjective evaluation results are shown in Figure 4. Selfassessments of the patients were consistent with the evaluation of the investigator. For wrinkles on the lateral canthus and neck, all of the subjects indicated slightly to very good improvement respectively (Figures 4A-4D). For wrinkles on the forehead and glabellum, just one subject indicated no change respectively. Other subjects indicated slightly to very good improvement (Figures 4B and 4D).
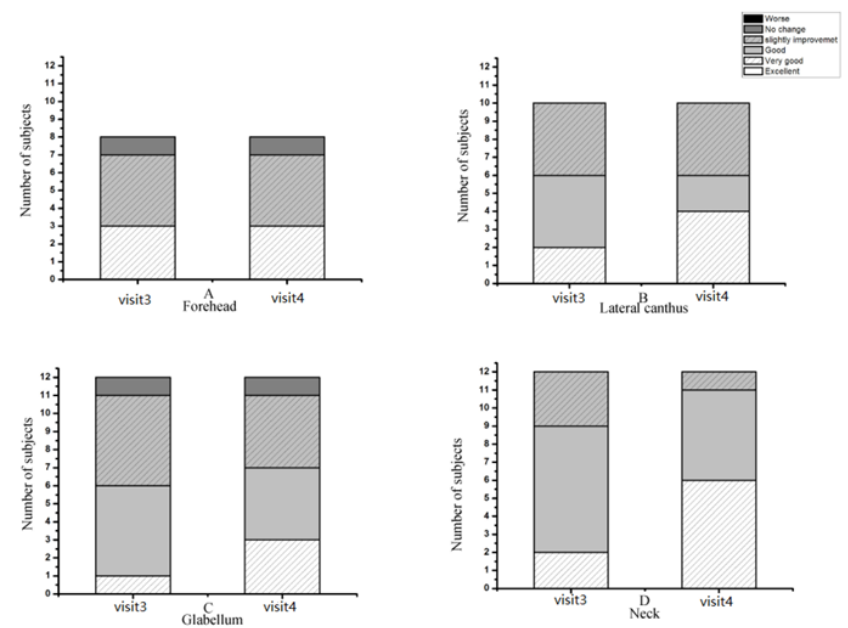

Figure 3. The objective evaluation of wrinkles 4 weeks after two sessions (visit 3, before irradiation) and 4 weeks after the last (third) treatment (visit 4, before irradiation). (A) Forehead; (B) Lateral canthus; (C) Glabellum; (D) Neck; Wrinkles on the forehead, lateral canthus, glabellum and neck were indicated high point improvement.
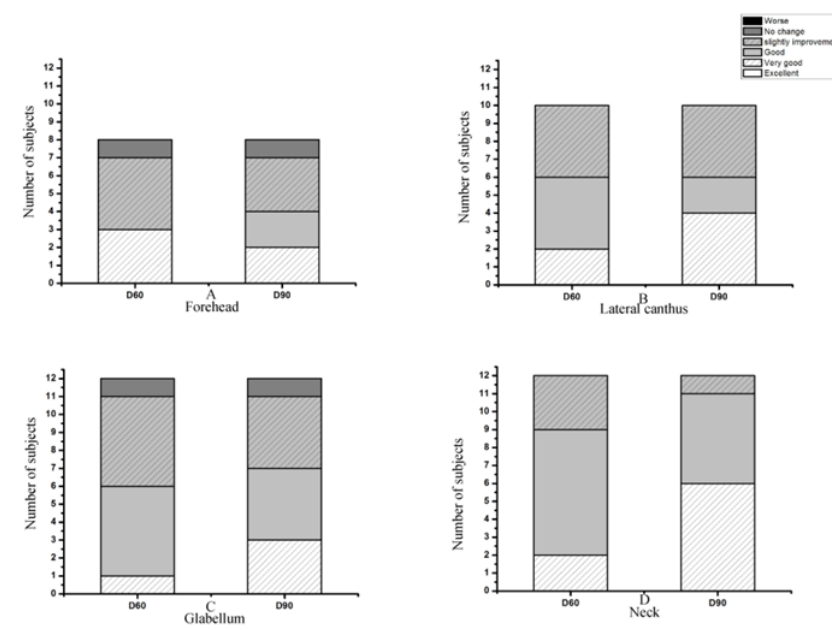

Figure 4. The results of subjective evaluation of wrinkles 4 weeks after two sessions (visit 3, before irradiation) and 4 weeks after the last (third) treatment (visit 4, before irradiation). (A) Forehead; (B) Lateral canthus; (C) Glabellum; (D) Neck. Similar improvements were observed in the subjective evaluation in each category.

\section{Adverse events}

During the treatment, all patients had transient mild erythema and edematous erythema after irradiation. The duration of side reactions is basically the same after each treatment. No other severe adverse reaction, such as pigmentary alterations, scarring, vesiculation, or infection associated with the treatment were observed. The mean pain score was 3.4 during the treatment Based on the 10-point VAS scale, and no severe or intolerable pain was reported. 


\section{Discussion}

With increasing life expectancy, symptoms of skin aging have gained considerable research attention. In the histopathology of aged skin, you would found a larger amount of elastincontaining fibrils accumulation in dermis, collagen synthesis decrease and abnormalities in the collagen fiber network. Clinically, these changes are predominantly characterized by the presence of wrinkles $[15,16]$.

Numerous methods are used to regain younger skin appearance. Cosmetic surgery is the current main treatment for skin rejuvenation, but this technique confers high risks and long downtime. Meanwhile, minimally invasive technologies, such as laser and RF, have gained popularity for skin treatment. The principle of RF is to convert rapidly changing currents into thermal energy [3,17]. The generated energy triggers the production of new collagen. RF has received higher degree of acceptance among Asians compared with lasers because the former easily penetrates into the skin without causing tissue diffraction or chromophore absorption [1].

RF devices are available exists in two forms: monopolar, bipolar. And our device is a special type of biopolar radiofrequency machine. Monopolar RF devices are equipped with an emission electrode and a large surface for collecting the electrode. Bipolar RF devices have two separated electrodes with a short distance in one applicator. Monopolar RF devices were the first to be approved by FDA for treatment of peri-orbital wrinkles $[17,18]$. In a monopolar system, the electrode, contacting with the skin, delivers current, and the large surface acts as the grounding pad for collecting the electrode [3]. In a bipolar system, the current transfers between two electrodes, and the depth of thermal damage generated is generally half the distance between the two electrodes [17]. Compared with bipolar devices, monopolar devices exhibit greater advantages in terms of the depth of penetration but also accompanied by higher incidence of side effects and potential for pain. As a special type of biopolar radiofrequency machine, FRF device uses an array of micro-needles arranged pairwise. Fractional photo-thermolysis RF devices create microscopic thermal damage zones, but the surrounding skin between the targeted areas are left intact and served as a reservoir of viable tissues; these devices will have less thermal damage and lower incidence of side effects.

Few randomized controlled trials were performed to examine the effects of FRF treatment on aging Asian skin. This study is the first to report the improvement of wrinkles on the neck by using this device. We found $87.5 \%, 80 \%, 100 \%$, and $100 \%$ improvements in skin wrinkles on the forehead, glabellum, lateral canthus, and neck, respectively, at visit 4 . These results are similar to those reported by Akita [19], who found that wrinkles on the lateral canthus significantly improved after treatment with FRF device. However, the effect of the treatment on wrinkles on the forehead was not significant. The difference outcomes may have originated from the differences in treatment programs and evaluation standards employed. Domyati, analyzed the effect of RF device on patients, and reported increased, epidermal thickness, granular layer thickness, and collagen content after the treatment [1]. Therefore, our results may be consistent with the expected outcome because wrinkles on different anatomic locations present similar effects after RF treatment.

Although high energy was used in some anatomic sites in this study, the treatment did not produce any systemic adverse events and/or permanent side effects. One study also found that the FRF technology only produced similar mild side effects such as erythema, swelling, scaling, and pain, which were tolerated [20]. These results are similar to those of the present study.

The main disadvantage of this work is the small number of subjects. As such, future studies must employ a larger sample size and a longer follow-up period to establish the long-term efficacy of FRF devices on skin aging. Overall, this study provides clinical experience for treatment of light skin aging in Asian population.

\section{References}

1. El-Domyati M, El-Ammawi TS, Medhat W, Moawad O, Brennan D, Mahoney MG, Uitto J. Radiofrequency facial rejuvenation: evidence-based effect. J Am Acad Dermatol 2011; 64: 524-535.

2. Lolis MS, Goldberg DJ. Radiofrequency in cosmetic dermatology: a review. Dermatol Surg 2012; 38: 1765-1776.

3. Alster TS, Lupton JR. Nonablative cutaneous remodeling using radiofrequency devices. Clin Dermatol 2007; 25: 487-491.

4. Lee HS, Lee DH, Won CH, Chang HW, Kwon HH, Kim $\mathrm{KH}$, Chung JH. Fractional rejuvenation using a novel bipolar radiofrequency system in Asian skin. Dermatol Surg 2011; 37: 1611-1619.

5. Atiyeh BS, Dibo SA. Nonsurgical nonablative treatment of aging skin: radiofrequency technologies between aggressive marketing and evidence-based efficacy. Aesthetic Plast Surg 2009; 33: 283-294.

6. Qin X, Li H, Jian X, Yu B. Evaluation of the efficacy and safety of fractional bipolar radiofrequency with highenergy strategy for treatment of acne scars in Chinese. J Cosmet Laser Ther 2015; 17: 237-245.

7. Khan MH, Sink RK, Manstein D, Eimerl D, Anderson RR. Intradermally focused infrared laser pulses: thermal effects at defined tissue depths. Lasers Surg Med 2005; 36: 270-280.

8. Manstein D, Herron GS, Sink RK, Tanner H, Anderson RR. Fractional photothermolysis: a new concept for cutaneous remodeling using microscopic patterns of thermal injury. Lasers Surg Med 2004; 34: 426-438.

9. Brightman L, Goldman MP, Taub AF. Sublative rejuvenation: experience with a new fractional radiofrequency system for skin rejuvenation and repair. $\mathrm{J}$ Drugs Dermatol 2009; 8: 9-13. 
10. Hruza G, Taub AF, Collier SL, Mulholland SR. Skin rejuvenation and wrinkle reduction using a fractional radiofrequency system. J Drugs Dermatol 2009; 8: 259-265.

11. Yeung CK, Chan NP, Shek SY, Chan HH. Evaluation of combined fractional radiofrequency and fractional laser treatment for acne scars in Asians. Lasers Surg Med 2012; 44: 622-630.

12. Ramesh M, Gopal M, Kumar S, Talwar A. Novel technology in the treatment of acne scars: the matrixtunable radiofrequency technology. J Cutan Aesthet Surg 2010; 3: 97-101.

13. Taub AF, Garretson CB. Treatment of acne scars of skin types II to $\mathrm{V}$ by sublative fractional bipolar radiofrequency and bipolar radiofrequency combined with diode laser. J Clin Aesthet Dermatol 2011; 4: 18-27.

14. Cho SI, Chung BY, Choi MG, Baek JH, Cho HJ, Park $\mathrm{CW}$, Lee $\mathrm{CH}$, Kim HO . Evaluation of the clinical efficacy of fractional radiofrequency microneedle treatment in acne scars and large facial pores. Dermatol Surg 2012; 38: 1017-1024.

15. El-Domyati M, El-Ammawi TS, Medhat W, Moawad O, Mahoney MG, Uitto J. Electro-optical synergy technique: a new and effective nonablative approach to skin aging. J Clin Aesthet Dermatol 2010; 3: 22-30.

16. Sukal SA, Geronemus RG. Thermage: the nonablative radiofrequency for rejuvenation. Clin Dermatol 2008; 26: 602-607.
17. Elsaie ML. Cutaneous remodeling and photorejuvenation using radiofrequency devices. Indian J Dermatol 2009; 54: 201-205

18. Alexiades-Armenakas M, Dover JS, Arndt KA. Unipolar versus bipolar radiofrequency treatment of rhytides and laxity using a mobile painless delivery method. Lasers Surg Med 2008; 40: 446-453.

19. Akita H, Sasaki R, Yokoyama Y, Negishi K, Matsunaga $\mathrm{K}$. The clinical experience and efficacy of bipolar radiofrequency with fractional photothermolysis for aged Asian skin. Exp Dermatol 2014; 23: 37-42.

20. Kaminaka C, Uede M, Matsunaka H, Furukawa F, Yamamoto Y. Clinical studies of the treatment of facial atrophic acne scars and acne with a bipolar fractional radiofrequency system. J Dermatol 2015; 42: 580-587.

\section{*Correspondence to}

Tong Lin

Institute of Dermatology

Chinese Academy of Medical Sciences and Peking Union Medical College

Cosmetic Lasers

Nanjing

PR China 